\title{
ANOMALOUS HIGHER BRANCHING PATTERN OF THE FEMORAL NERVE: A CASE REPORT WITH CLINICAL IMPLICATIONS
}

\author{
Srijit Das ${ }^{1}$, Neelam Vasudeva ${ }^{2}$ \\ University Kebangsaan Malaysia, Kuala Lumpur, Malaysia: Department of Anatomy'; Maulana Azad Medical College, \\ Bahadur Shah Zafar Marg, New Delhi, India: Department of Anatomy² \\ Summary: The femoral nerve usually divides into anterior and posterior branches below the inguinal ligament. In the pre- \\ sent case, we report the anomalous higher branching pattern of the femoral nerve on both sides of a 52 year male cada- \\ ver. The femoral nerve divided into the anterior and posterior branches above the inguinal ligament. Such a higher division \\ of femoral nerve is a rare finding and it may be important for surgeons, orthopaedicians and anaesthetists in day to day \\ clinical practice.
}

Key words: Femoral nerve; Branches; Anomaly; Variation; Nerve block; Failure

\section{Introduction}

Orthopaedic and plastic surgeons perform the femoral nerve block for providing anaesthesia to anterior and lateral aspects of the thigh. The femoral nerve may often be injured by suture or staples, tissue scar entrapment, local anesthesia blockade or direct compression $(5,8)$. Conventionally, the femoral nerve is known to divide into anterior and posterior branches below the inguinal ligament (10, 11). The femoral nerve block is performed on the main stem of the nerve, inferior to the inguinal ligament, before it divides into anterior and posterior branches (3).

In the present study, we report an anomalous higher branching pattern of the femoral nerve above the inguinal ligament, which is a rare finding. Any anomalous position of the femoral nerve or its higher level of branching pattern as seen in the present case may confuse any orthopaedic surgeon who would usually perform the nerve block below the inguinal ligament, thereby blocking only one of its branches with the other branch intact, thereby resulting in incomplete femoral nerve block. Prior anatomical knowledge of the normal as well as the abnormal anatomy of the femoral nerve and its branching pattern may be clinically important for anaesthetists, orthopaedicians and surgeons, in day to day clinical practice.

\section{Case report}

During a routine dissection of a cadaver for an undergraduate teaching programme, we detected an anomalous branching pattern of the femoral nerve on both sides of a 52 year male cadaver. The course of the femoral nerve and its relations to other structures were studied in detail and an appropriate photograph was taken (Fig. 1).

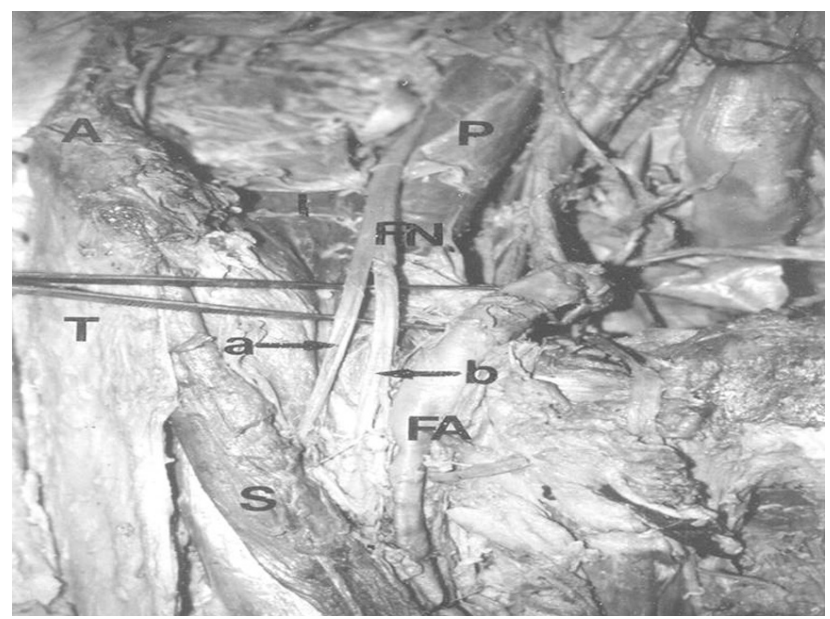

Fig. 1: Photograph of dissected right leg (anterior view) showing: P - Psoas muscle; I - Iliacus muscle; FN Femoral nerve; a - Anterior branch of the femoral nerve (innervating sartorius muscle); b - Posterior branch of the femoral nerve; A - Anterior superior iliac spine; T - Tensor fasciae latae; $\mathrm{S}$ - Sartorius muscle. The upper limb of the forceps marks the region of the inguinal ligament above which the femoral nerve has divided.

\section{Observations}

In both the lower limbs, the femoral nerve was observed to originate from the posterior division of the ventral rami 
of the second to fourth lumbar spinal nerves. The femoral nerve ('FN' in Fig. 1) descended through the psoas major muscle ('P' in Fig. 1) and $3.2 \mathrm{~cm}$ above the midpoint of the inguinal ligament and divided into two branches, i.e. the anterior ('a' in Fig. 1) and the posterior ('b' in Fig. 1). The anterior branch further gave the intermediate cutaneous nerve of thigh, medial cutaneous nerve of thigh and the branch to the sartorius muscle. The saphenous nerve and the branch to the quadriceps femoris muscle were found to arise from the posterior branch. The posterior branch was related laterally to the femoral artery ('FA' in Fig. 1). No other abnormalities were noted.

\section{Discussion}

As per standard textbooks of anatomy, the femoral nerve is the largest branch of the lumbar plexus and it arises from the posterior division of the anterior (ventral) rami of the second, third and fourth lumbar nerves $(10,11)$. The femoral nerve descends on the psoas major muscle and enters the thigh deep to the inguinal ligament at the lateral edge of the femoral sheath, which separates it from the femoral artery (10). A branch to the pectineus muscle is given off as the femoral nerve enters the femoral triangle. Distally to the inguinal ligament, the femoral nerve divides into two branches: the anterior and the posterior one $(10,11)$. The branches from the anterior divisions are mainly to the sartorius muscle and two cutaneous branches, i.e. the medial cutaneous nerve of thigh and the intermediate cutaneous branch of thigh. The saphenous nerve, nerve to quadriceps femoris and branch to knee joint are the branches from the posterior division (11).

The exact knowledge of topographical anatomy of the femoral nerve is required for any successful femoral nerve block. For this procedure, first the inguinal ligament is located, the femoral artery is palpated $2-3 \mathrm{~cm}$ below the ligament and a needle is passed just laterally to the femoral artery (2). Thus, the femoral nerve is always easy to block inferior to the inguinal ligament (3). The ideal procedure is to pass a needle $1-2 \mathrm{~cm}$ laterally to the femoral artery with a slight cephalad angulation and push $10 \mathrm{ml}$ of $2 \%$ procaine or equivalent anaesthetic solution $(2,3)$.

At times, when the femoral nerve has already divided above the inguinal ligament, there are chances that both the branches, i.e. the anterior and the posterior branch, may be found deep and inferior to the inguinal ligament. Interestingly, any attempt to perform the femoral nerve block may involve only one of its branches, with the other one escaping the nerve block procedure. Either of the two branches, i.e. the anterior or the posterior, may be blocked, with the resultant effect of incomplete or partial anaesthesia. This may be a difficult situation for the orthopaedician and the surgeon who may not be aware of the fact that the failure of anaesthesia is due to the anomalous branching pattern of the femoral nerve, i.e. branching well above the inguinal ligament.

A thorough search of the literature reveals that there have been various reports on the variations of the lateral femoral cutaneous nerves and the lumbar plexus $(1,4,7,9)$. The reasons why the anatomy of lateral cutaneous femoral nerve has also been always dealt with in detail is because of the fact that it forms an excellent landmark to check any iatrogenic injury (6).

There is a paucity of literature on the abnormal or higher branching pattern of the femoral nerve. We, as anatomists, definitely feel that this anomaly would be of assistance to the anaesthetists, orthopaedicians and surgeons, who go by the conventional knowledge of anatomy regarding the exact site for performing femoral nerve block. To the best of our knowledge, the higher branching pattern of the femoral nerve above the inguinal nerve has not been reported in any other past study and this knowledge may be clinically important for anesthetists, orthopaedicians and surgeons operating on the leg.

\section{References}

1. Aszmann OC, Dellon ES, Dellon AL. Anatomical course of the lateral femoral cutaneous nerve and its susceptibility to compression and injury. Plast Reconstr Surg 1997;100:600-4

2. Collins VJ. Principles of Anesthesiology. General and regional anesthesia. $3^{\text {rd }}$ ed. Pennsylvania: Lea \& Febiger, 1993: pp-1395-97.

3. Ellis H, Feldman S, Harrop Griffith W (2004). Anatomy for anaesthetists. Massachusetts, Blackwell publishing,2004: pp-188-191.

4. Erbil KM, Sargon FM, Sen F, Ozturk H, Tascioglu B, Yemen N, Ozozan VO Examination of the variations of lateral femoral cutaneous nerves: report of two cases. Anat Sci Int 2002;77:247-9.

5. Garcia-Urena MA, Vega V, Rubio G, Velasco MA. The femoral nerve in the repair of inguinal hernia: well worth remembering. Hernia 2005;9:384-7.

6. Grothaus MC, Holt M, Mekhail AO, Ebraheim NA, Yeasting RA. Lateral femoral cutaneous nerve: an anatomic study. Clin Orthop Relat Res 2005;437:164-8.

7. Hospodar PP, Ashman ES, Traub JA. Anatomic study of the lateral femoral cutaneous nerve with respect to the ilioinguinal surgical dissection. J Orthop Trauma 1999;13:17-9.

8. Kim DH, Murovic JA, Tiel RL, Kline DG. Intrapelvic and thigh-level femoral nerve lesions: management and outcomes in 119 surgically treated cases. J Neurosurg 2004;100:989-96.

9. Mine Erbil K, Onderoglu S, Basar R. Unusual branching in lumbar plexus. Case report. Folia Morphol (Warsz) 1998;57:377-81.

10. Sinnatamby CS (2001). Last's Anatomy. Regional and Applied. London: Churchill Livingstone, 2001: pp-115.

11. Standring S (2005). Gray's Anatomy. The Anatomical Basis of Clinical Practice. $38^{\text {th }}$ ed. New York: Elsevier Churchill Livingstone, pp-1403, 1413 \& 1455.

\section{Corresponding author:}

Submitted August 2007. Accepted September 2007.

Dr. Neelam Vasudeva, MBBS, MS, Maulana Azad Medical College, Bahadur Shah Zafar Marg, New Delhi-110002, India, e-mail: vasudevavn@yahoo.co.in 